\title{
FLAVONOIDS FROM THE LEAVES OF BAMBUSA PERVARIABILIS MCCLURE
}

\author{
JIA SUN, YONG-DE YUE ${ }^{\prime *}, F E N G$ TANG, XUE-FENG GUO
}

SFA Key Open Laboratory on Bamboo and Rattan Science and Technology, International Centre for Bamboo and Rattan, Beijing 100102, China

(Received: December 29, 2009 - Accepted: July 6, 2010)

\begin{abstract}
A new flavonoid, 5,4'-dihydroxy-3',5'-dimethoxy-7-O[ $\beta$-D-apiose- $(1 \rightarrow 2)]-\beta$-D-glucopyranosyl flavonoside(1), along with four known flavonoids $5,7,3^{\prime}, 4^{\prime}$ '-tetrahydroxy-6-C- $\beta$-L-arabinosyl flavonoside(2), 5,7,4'-trihydroxy-6-C- $\beta$-D- glucopyranosyl flavonoside(3), 5,7,3',4'-tetrahydroxy-6-C- $\beta$-Dglucopyranosyl flavonoside(4), 5,7,3',4'-tetrahydroxy-8-C- $\beta$-D-glucopyranosyl flavonoside(5) were isolated from the leaves of Bambusa pervariabilis McClure. Their structures were determined on the basis of spectroscopic techniques and chemical methods.
\end{abstract}

Keywords: Bambusa pervariabilis McClure; flavonoids; 5,4'-dihydroxy-3',5'-dimethoxy-7-O[ $\beta$-D-apiose-(1 $\rightarrow 2)]$ - $\beta$-D- glucopyranosyl flavonoside

\section{INTRODUCTION}

Flavonoids, important active components of plant, showed significant anti-inflammatory and antioxidant activities, and had the effects of treating cardiovascular disease $^{1-3}$ and different types of cancer-preventive activity ${ }^{4}$ ${ }^{5}$. Phytochemical research on bamboo leaves showed that they were rich in flavonoids ${ }^{6-8}$. Consequently, flavonoids extracted from bamboo leaves have been used as health products and food additive. $B$. pervariabilis McClure is one of the bamboo species in China. Chinese people realized the medical and health care effects of $B$. pervariabilis McClure leaf long ago, and used it as a traditional medicine to treatment of febrile disorder, exogenous diseases, cooling blood and Hemostasis ${ }^{9}$. Our previous research exhibited that the presence of coumarins in the leaves of $B$. pervariabilis $\mathrm{McClure}^{10}$. Extensive chromatography of the EtOH extract of the leaves of B. pervariabilis McClure had led to the isolation of a new flavonoid and four known flavonoids reported from this species for the first time. This paper deals with the isolation and structural elucidation of the new compound $\mathbf{1}$.

\section{EXPERIMENTAL}

\section{General experimental procedures}

Melting points were determined with Shenguang WRX-1S thermal values analyzer with microscope and are uncorrected. The optical rotation was measured with a Perkin-Elmer 343 Polarimeter. UV spectra were obtained on Waters 2695 HPLC with photodiode array detector. IR spectra were taken on a Thermo Nicolet FT-IR NEXUS 670 spectrophotometer with KBr pellets. NMR spectra were recorded on Varian System-600. HRESIMS spectra were performed on AutoSpec Ultima-TOF mass spectrometer and ESIMS data were obtained with an Agilent 1100 Series mass spectrometer.

Plant material

The leaves of $B$. pervariabilis McClure were collected from Nanning City, Guangxi Province, China in September 2008, and identified by Professor Dayong Huang, Bamboo Research Institute, Nanning Academy of Forestry,
Nanning, China. A voucher specimen (200810-01) is deposited at the International Centre for Bamboo and Rattan (ICBR), Beijing, China.

Extraction and isolation

The shade-dried leaves of $B$. pervariabilis McClure $(8.24 \mathrm{~kg})$ were extracted with $95 \%$ EtOH by cold percolation for three times. A residue of $765.3 \mathrm{~g}$ was obtained after removal of the solvent by evaporation. The residue was suspended in $\mathrm{H}_{2} \mathrm{O}$ and extracted with petroleum ether. The fraction after being extracted with petroleum ether was subjected to macroporous absorption resin (AB-8) and eluted with $\mathrm{H}_{2} \mathrm{O}, 20 \% \mathrm{EtOH}, 40 \% \mathrm{EtOH}, 60 \% \mathrm{EtOH}, 80 \%$ $\mathrm{EtOH}$, and acetone. The $40 \% \mathrm{EtOH}$ fraction $(42.9 \mathrm{~g}$ ) was then chromatographed over Sephadex LH-20 and eluted with $\mathrm{MeOH}$ repeatedly, to yield compound 1 (123.2 mg), compounds 2 (996.8 mg), compounds 3 (812.0mg), and compounds $4(196.0 \mathrm{mg})$ and $5(137.6 \mathrm{mg})$.

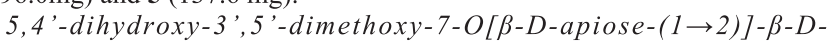
glucopyranosyl flavonoside(1)

Yellow amorphous powder $(\mathrm{MeOH}), \operatorname{mp} 250.6-252.4^{\circ} \mathrm{C} ;[\alpha]_{D}^{\mathcal{Z}}=-77^{\circ}(\mathrm{c}$ $=0.1$, DMSO) ; UV 1 max (nm): 251.0, 273.5 and 352.2, FT-IR (KBr) 1 max $\left(\mathrm{cm}^{-1}\right): 3266.4,2917.2,1651.2,1596.9,1571.5,1493.1,1455.4 ;{ }^{1} \mathrm{H}$ and ${ }^{13} \mathrm{C}$ NMR data (Table 1); HRESIMS, $m / z$ 647.1557 [M+Na] ${ }^{+} \mathrm{C}_{28} \mathrm{H}_{32} \mathrm{NaO}_{16}{ }^{+}$(calcd for 647.1588); Negative ion ESIMS $m / z 659.2[\mathrm{M}+\mathrm{Cl}]$.

\section{RESULTS AND DISCUSSION}

Compound 1 was obtained as a yellow amorphous powder, mp 250.6$252.4^{\circ} \mathrm{C},[\alpha]_{D}^{\mathcal{B}}=-77^{\circ}\left(c=0.1\right.$, DMSO). The molecular formula, $\mathrm{C}_{28} \mathrm{H}_{32} \mathrm{O}_{16}$, was deduced from the negative HRESIMS at $m / z 647.1557[\mathrm{M}+\mathrm{Na}]^{+} \mathrm{C}_{28} \mathrm{H}_{32} \mathrm{NaO}_{16}{ }^{+}$ (calcd for 647.1588).The ESIMS (negative) showed ion peaks at $\mathrm{m} / \mathrm{z} 659.2$ $[\mathrm{M}+\mathrm{Cl}]^{-}$. It was recognized as a flavonoid from a positive test with $\mathrm{Mg}-\mathrm{HCl}$ and Molish reagents. The IR spectrum displayed characteristic absorption bands for hydroxyl $\left(3266.4 \mathrm{~cm}^{-1}\right)$, methyl $(2917.2)$, carbonyl $\left(1651.2 \mathrm{~cm}^{-1}\right)$, and aromatic rings $\left(1596.9 \mathrm{~cm}^{-1}, 1571.5 \mathrm{~cm}^{-1}, 1493.1 \mathrm{~cm}^{-1}, 1454.4 \mathrm{~cm}^{-1}\right)$. The UV spectrum showed absorption maxima at $251.0,273.5$ and $352.2 \mathrm{~nm}$, characteristic for the Flavonoid. 
Table 1. NMR spectral data (d, ppm) for compound (1) in DMSO- $\mathrm{d}_{6}{ }^{\mathrm{a}}$.

\begin{tabular}{|c|c|c|c|c|c|c|c|c|c|c|c|}
\hline \multirow[b]{2}{*}{ No. } & \multicolumn{8}{|c|}{ Aglycone moirty } & \multicolumn{3}{|c|}{ gar moiety } \\
\hline & $\delta_{\mathrm{H}}$ & $\delta_{\mathrm{OH}}$ & $\delta_{\mathrm{c}}$ & DEPT & $\begin{array}{l}{ }^{1} \mathrm{H}-{ }^{-1} \mathrm{H} \\
\mathrm{COSY}\end{array}$ & No. & $\delta_{\mathrm{H}}$ & $\delta_{\text {он }}$ & $\delta_{\mathrm{c}}$ & DEPT & ${ }^{1} \mathrm{H}-{ }^{-1} \mathrm{H} \operatorname{COSY}$ \\
\hline 2 & & & 164.3 & $\mathrm{C}$ & & D-glucuse & & & & & \\
\hline 3 & $7.06(1 \mathrm{H}, \mathrm{s})$ & & 104.0 & $\mathrm{CH}$ & & $1 "$ & $5.07(1 \mathrm{H}, \mathrm{d}, 9.5)$ & & 98.4 & $\mathrm{CH}$ & H-2" \\
\hline 4 & & & 182.2 & $\mathrm{C}$ & & $2 "$ & $3.49(1 \mathrm{H}, \mathrm{m})$ & & 76.9 & $\mathrm{CH}$ & $\mathrm{H}-1 "$ \\
\hline 5 & & & 161.2 & $\mathrm{C}$ & & $2 "-\mathrm{OH}$ & & $5.34 \mathrm{brs}$ & & & \\
\hline $5-\mathrm{OH}$ & & $12.95 \mathrm{~s}$ & & & & $3 "$ & $3.71(1 \mathrm{H}, \mathrm{m})$ & & 76.2 & $\mathrm{CH}$ & H-2",H-4" \\
\hline 6 & $6.43(1 \mathrm{H}, \mathrm{s})$ & & 99.6 & $\mathrm{CH}$ & & $3 "-\mathrm{OH}$ & & $5.13 \mathrm{brs}$ & & & \\
\hline 7 & & & 162.8 & $\mathrm{C}$ & & $4 "$ & $3.19(1 \mathrm{H}, \mathrm{m})$ & & 70.0 & $\mathrm{CH}$ & H-3",H-5" \\
\hline 8 & $6.91(1 \mathrm{H}, \mathrm{s})$ & & 95.4 & $\mathrm{CH}$ & & $5 "$ & $3.53(1 \mathrm{H}, \mathrm{m})$ & & 77.3 & $\mathrm{CH}$ & H-4",H-6" \\
\hline 9 & & & 157.0 & $\mathrm{C}$ & & $6 "$ & $\begin{array}{l}3.71(1 \mathrm{H}, \mathrm{m}) \\
3.59(1 \mathrm{H}, \mathrm{m})\end{array}$ & & 60.7 & $\mathrm{CH}_{2}$ & H-5",H-6" \\
\hline 10 & & & 105.5 & $\mathrm{C}$ & & $6 "-\mathrm{OH}$ & & $4.69 t(5.9)$ & & & \\
\hline 1 & & & 120.3 & $\mathrm{C}$ & & D-apiose & & & & & \\
\hline 2' & $7.34(1 \mathrm{H}, \mathrm{s})$ & & 104.6 & $\mathrm{CH}$ & & 1 "” & $5.30(1 \mathrm{H}, \mathrm{d}, 2.1)$ & & 108.8 & $\mathrm{CH}$ & $\mathrm{H}-2$ '” \\
\hline $3^{\prime}$ & & & 148.3 & $\mathrm{C}$ & & $2 " ”$ & $3.74(1 \mathrm{H}, \mathrm{m})$ & & 75.9 & $\mathrm{CH}$ & $\mathrm{H}-1$ '” \\
\hline 3'- $-\mathrm{OCH}_{3}$ & $3.87(3 \mathrm{H}, \mathrm{s})$ & & 56.5 & $\mathrm{CH}_{3}$ & & 2"'-OH & & $5.15 \mathrm{brs}$ & & & \\
\hline $4^{\prime}$ & & & 140.1 & $\mathrm{C}$ & & $3 " '$ & & & 79.4 & $\mathrm{C}$ & \\
\hline 4'-OH & & $9.37 \mathrm{~s}$ & & & & 3"'-OH & & 4.63brs & & & \\
\hline 5 ' & & & 148.3 & $\mathrm{C}$ & & $4 " '$ & $\begin{array}{l}3.64(1 \mathrm{H}, \mathrm{d}, 9.5) \\
3.90(1 \mathrm{H}, \mathrm{d}, 9.5)\end{array}$ & & 74.1 & $\mathrm{CH}_{2}$ & $\mathrm{H}-4$ "' \\
\hline $5^{\prime}-\mathrm{OCH}_{3}$ & $3.87(3 \mathrm{H}, \mathrm{s})$ & & 56.5 & $\mathrm{CH}_{3}$ & & $5 " ”$ & $\begin{array}{l}3.28(1 \mathrm{H}, \mathrm{m}) \\
3.34(1 \mathrm{H}, \mathrm{m})\end{array}$ & & 64.3 & $\mathrm{CH}_{2}$ & $\mathrm{H}-5$ "” \\
\hline $6^{\prime}$ & $7.34(1 \mathrm{H}, \mathrm{s})$ & & 104.6 & $\mathrm{CH}$ & & 5"'-OH & & $4.65 \mathrm{brs}$ & & & \\
\hline
\end{tabular}

${ }^{\text {a The }}{ }^{1} \mathrm{H}$ and ${ }^{13} \mathrm{C}$ NMR spectral data were measured at $600 \mathrm{MHz}$, and the $J$ values (parentheses) are in hertz.

Besides the characteristic signal for A,C-rings of the flavone skeleton at $\delta 6.43(1 \mathrm{H}, \mathrm{s}) \delta 6.91(1 \mathrm{H}, \mathrm{s})$, the $1 \mathrm{H}$ NMR spectrum of compound 1 displayed signals of a $1^{\prime}, 3^{\prime}, 4^{\prime}, 5^{\prime}$ tetra-substituted phenyl moiety(B-ring) at $7.34(2 \mathrm{H}, \mathrm{s})$ and two methoxyl groups at $\delta 3.87(6 \mathrm{H}, \mathrm{s})$, as well as five oxygenated proton signals at $\delta 3.19-3.74$, and three methylene group signals at $\delta 3.28-3.90$. Acid hydrolysis of compound $\mathbf{1}$ affording glucose and apiose indicated that the glucose and apiose were linked to the aglycone with $\mathrm{C}-\mathrm{O}$ bond. Two anomeric proton signals at $\delta 5.07(1 \mathrm{H}, \mathrm{d}, J=9.5 \mathrm{~Hz})$ and $\delta 5.30(1 \mathrm{H}, \mathrm{d}, J=2.1 \mathrm{~Hz})$ suggested the presence of two sugar units in compound $\mathbf{1}$, which were identified as glucose and apiose by ${ }^{13} \mathrm{C}$ NMR and ${ }^{1} \mathrm{H}$ NMR. In the DEPT spectrum (Table1), two methylene group signals $\delta(\mathrm{C}) 60.7$ and $\delta(\mathrm{C}) 64.3$ were the signals of sugarend. The anomeric proton signals at $\delta 5.07(1 \mathrm{H}, \mathrm{d}, J=9.5 \mathrm{~Hz})$ was identified as glucose. The anomeric proton signals at $\delta 5.30(1 \mathrm{H}, \mathrm{d}, J=2.1 \mathrm{~Hz})$ was identified as apiose. This assignment was also proved by the ${ }^{13} \mathrm{C}$ NMR signals at $\delta(\mathrm{C})$ 108.8 , which was the characteristic signal of anomeric carbon of $\mathrm{O}$-apiose, the signals of $\delta(\mathrm{C}) 74.1$ was the C-4' of apiose and the quaternary carbon signals $\delta(\mathrm{C}) 79.4$ was also confirmed the apiose was existed in the flavone. In the HMBC spectrum (Figure 2), long-range correlations from $\mathrm{H}-1$ of glucose to $\mathrm{C}-7$ of the flavone nucleus showed that the glucose was linked to C-O-C of the flavone nucleus and long-range correlations from $\delta 5.30(1 \mathrm{H}, \mathrm{d}, J=2.1 \mathrm{~Hz})$ to $\mathrm{C}-2$ $\delta(\mathrm{C}) 76.9$ of the glucose nucleus and $\delta 3.49(1 \mathrm{H}, \mathrm{m})$ to $\delta(\mathrm{C}) 108.8$ of the apiose nucleus showed the apiose was linked to $\mathrm{C}-2$ of the glucose nucleus by $\mathrm{C}-\mathrm{O}-\mathrm{C}$ bond., the chemical shift of the glucose $\mathrm{C}-2 \delta(\mathrm{C}) 76.9$ moved to down-field about $4 \mathrm{ppm}$, the assignment was also proved the apiose was linked to $\mathrm{C}-2$ of the glucose. All the proton and carbon signals were assigned by HSQC, DEPT, ${ }^{1} \mathrm{H}-{ }^{-} \mathrm{HCOSY}$ and HMBC experiments. The coupling constants of anomeric protons indicated that glucosyl and apiosyl linkage were $\beta$-configuration ${ }^{11}$, ${ }^{12}$. This assignment was also proved by the significant NOESY correlations (Figure 3). On the basis of above evidence, the structure of compound 1 was determined as 5,4'-dihydroxy-3',5'-dimethoxy-7-O[ $\beta$-D-apiose-( $(1 \rightarrow 2)]-\beta-D-$ glucopyranosyl flavonoside.

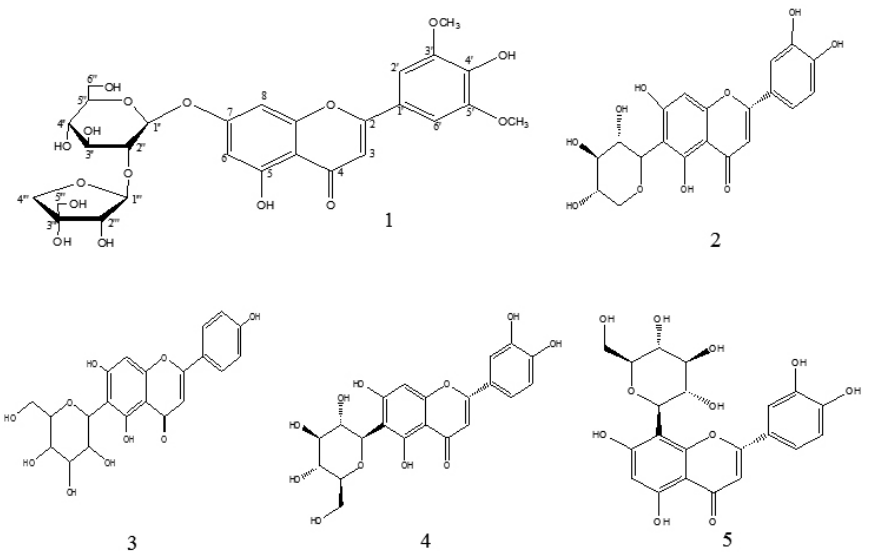

Figure 1. Structures of 1-5 


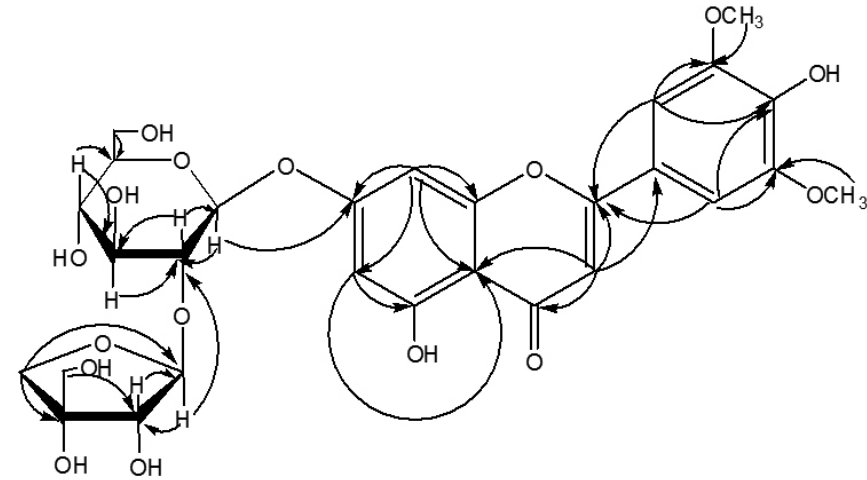

Figure 2. Significant HMBC correlations of compound (1)

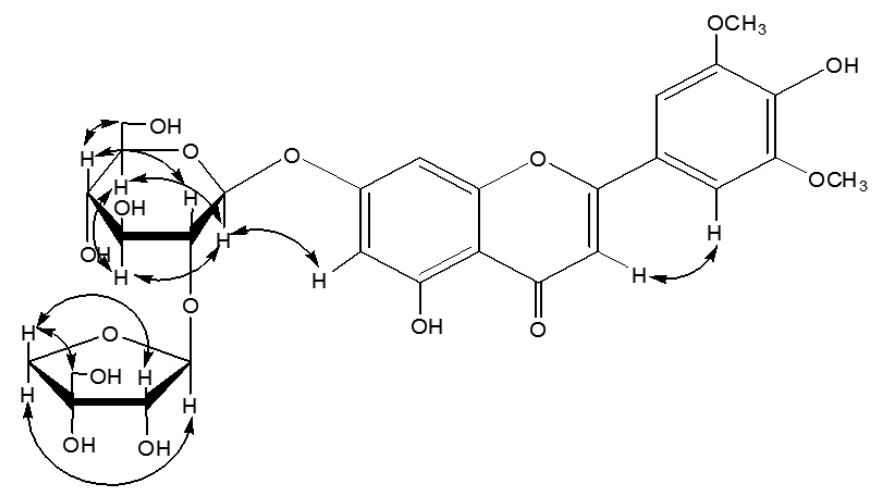

Figure 3. Significant NOESY correlations of compound (1)

5,7,3',4'-tetrahydroxy-6-C- $\beta$-L-arabinosyl flavonoside(2). Yellow amorphous powder, mp $226.2-227.0^{\circ} \mathrm{C} .{ }^{1} \mathrm{H}$ NMR $\left(600 \mathrm{MH}_{\mathrm{Z}}, \mathrm{MeOD}_{4}\right.$, $\delta, \quad \mathrm{ppm}, \quad \mathrm{J} / \mathrm{Hz}): \quad 6.806(\mathrm{~s}, 1 \mathrm{H}), \quad 6.405(\mathrm{~s}, 1 \mathrm{H}), \quad 7.276(\mathrm{~s}), \quad 6.806,6.793(\mathrm{~d}, 1 \mathrm{H})$, 7.276,7.290(d,1H), 4.692,4.708(d,1H), 3.863,3.859(m,1H), $3.501,3.507,3.517,3.522(\mathrm{q}, 1 \mathrm{H}), \quad 4.126,4.142,4.158(\mathrm{t}, 1 \mathrm{H}), \quad 3.631,3.611(\mathrm{~d}, 1 \mathrm{H}$ )3.895,3.899,3.916,3.919(q,1H). ${ }^{13} \mathrm{C}$ NMR (600 MHZ, MeOD-d $, \delta, \mathrm{ppm}, \mathrm{J} /$ $\mathrm{Hz}): 166.36(\mathrm{C}), 103.91(\mathrm{CH}), 184.04(\mathrm{C}), 158.75(\mathrm{C}), 109.30(\mathrm{C}), 164.79(\mathrm{C})$, 95.59(CH), $158.75(\mathrm{C}), \quad 105.17(\mathrm{C}), \quad 123.75(\mathrm{C}), \quad 114.14(\mathrm{CH}), \quad 147.06(\mathrm{C})$, 151.06(C), 116.79(CH), 120.34(CH), 76.22(CH), 70.74(CH), 75.85(CH), $70.83(\mathrm{CH}), 71.89\left(\mathrm{CH}_{2}\right)$.

5,7,4'-trihydroxy-6-C- $\beta$-D-glucopyranosyl flavonoside(3). Yellow amorphous powder, mp 187.3--188.6 ${ }^{\circ} \mathrm{C} .{ }^{1} \mathrm{H}$ NMR $\left(600 \mathrm{MH}_{3}, \mathrm{MeOD}^{-} \mathrm{d}_{4}, \delta\right.$, $\mathrm{ppm}, \mathrm{J} / \mathrm{Hz}): 6.532(\mathrm{~s}, 1 \mathrm{H}), 6.435(\mathrm{~s}, 1 \mathrm{H}), 6.854,6.868(\mathrm{~d}, 2 \mathrm{H}), 7.779,7.765(\mathrm{~d}, 2 \mathrm{H})$, $3.414,3.404(\mathrm{~d}, 1 \mathrm{H}), \quad 4.834,4.851(\mathrm{~d}, 1 \mathrm{H}), \quad 4.124,4.110,4.096(\mathrm{t}, 1 \mathrm{H})$, $3.429,3.420(\mathrm{~d}, 1 \mathrm{H}), \quad 3.364-3.351(\mathrm{~m}, 1 \mathrm{H}), \quad 3.828,3.810(\mathrm{~d}, 1 \mathrm{H}) 3.698,3.689,3.6$ 78,3.669(q,1H). ${ }^{13} \mathrm{C}$ NMR (600 MHZ, MeOD-d, $\left.\delta, \mathrm{ppm}, \mathrm{J} / \mathrm{Hz}\right): 166.18(\mathrm{C})$, 103.87(CH), $184.04(\mathrm{C}), \quad 158.75(\mathrm{C}), \quad 109.25(\mathrm{C}), \quad 165.14(\mathrm{C}), 95.29(\mathrm{CH})$, 162.83(C), 105.15(C), 123.12(C), $129.45(\mathrm{CH}), \quad 117.06(\mathrm{CH}), \quad 162.06(\mathrm{C})$, 117.06(CH), 129.45(CH), 80.15(CH), 75.31(CH), 72.59(CH), 71.99(CH), $82.64(\mathrm{CH}), 62.87\left(\mathrm{CH}_{2}\right)$.

5,7,3', 4'-tetrahydroxy-6-C- $\beta$-D-glucopyranosyl flavonoside(4). Yellow amorphous powder, mp $213.7-214.5^{\circ} \mathrm{C} .{ }^{1} \mathrm{H}$ NMR $\left(600 \mathrm{MH}_{\mathrm{z}}, \mathrm{MeOD}-\mathrm{d}_{4}, \delta\right.$, ppm, J/Hz): 6.663(s,1H), 6.474(s,1H), $7.391(\mathrm{~s}, 1 \mathrm{H}), \quad 6.888,6.872(\mathrm{~d}, 1 \mathrm{H})$, $7.420,7.416,7.403,7.399(\mathrm{q}, 1 \mathrm{H}), \quad 3.201,3.184(\mathrm{~d}, 1 \mathrm{H}), \quad 4.580,4.560(\mathrm{~d}, 1 \mathrm{H})$, $3.141,3.122,3.104,3.086(\mathrm{q}, 1 \mathrm{H}), 4.047,4.028,4.010(\mathrm{t}, 1 \mathrm{H}), 3.167,3.157(\mathrm{~m}, 1 \mathrm{H})$, $3.684,3.662(\mathrm{~d}, 1 \mathrm{H}) \quad 3.535,3.497,3.442,3.409,3.397,3.385,3.373(\mathrm{~m}, 1 \mathrm{H}) .{ }^{13} \mathrm{C}$

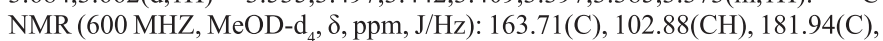
$160.77(\mathrm{C}), \quad 108.95(\mathrm{C}), \quad 163.32(\mathrm{C}), \quad 93.54(\mathrm{CH}), \quad 156.25(\mathrm{C}), \quad 103.48(\mathrm{C})$, $121.50(\mathrm{C}), 113.38(\mathrm{CH}), 145.82(\mathrm{C}), 149.76(\mathrm{C}), 116.12(\mathrm{CH}), 119.04(\mathrm{CH})$, 79.02(CH), 73.10(CH), 70.70(CH), 70.25(CH), 81.67(CH), 61.57( $\left.\mathrm{CH}_{2}\right)$.
5,7,3',4'-tetrahydroxy-8-C- $\beta$-D-glucopyranosyl flavonoside(5). Yellow amorphous powder, $\mathrm{mp} 237.5-238.9^{\circ} \mathrm{C} .{ }^{1} \mathrm{H}$ NMR $\left(600 \mathrm{MH}_{\mathrm{Z}}, \mathrm{MeOD}_{\mathrm{d}}\right.$, $\delta, \mathrm{ppm}, \mathrm{J} / \mathrm{Hz}): 6.537(\mathrm{~s}, 1 \mathrm{H}), 6.156(\mathrm{~s}, 1 \mathrm{H}), \quad 7.369(\mathrm{~d}, 1 \mathrm{H}), \quad 6.763,6.737(\mathrm{~d}, 1 \mathrm{H})$, 7.434,7.408,7.281(q,1H), $3.135(\mathrm{~d}, 1 \mathrm{H}), \quad 4.615,4.577(\mathrm{~d}, 1 \mathrm{H}), \quad 3.233(\mathrm{q}, 1 \mathrm{H})$, $3.709(\mathrm{~d}, 1 \mathrm{H}), 3.055(\mathrm{~m}, 1 \mathrm{H}), 3.576(\mathrm{~d}, 1 \mathrm{H}) ; 3.436(\mathrm{~m}, 1 \mathrm{H}) .{ }^{13} \mathrm{C}$ NMR (600 MHZ, MeOD-d $, \delta, p p m, J / H z): 164.54(\mathrm{C}), 102.87(\mathrm{CH}), 182.47(\mathrm{C}), 160.84(\mathrm{C})$, $98.57(\mathrm{CH}), \quad 162.99(\mathrm{C}), \quad 105.01(\mathrm{C}), \quad 156.45(\mathrm{C}), \quad 104.50(\mathrm{C}), \quad 122.49(\mathrm{C})$, 113.38(CH), 146.25(C), 150.05(C), 116.12(CH), $119.82(\mathrm{CH}), 79.23(\mathrm{CH})$,

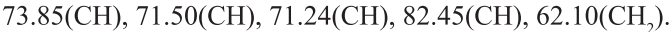

Furthermore, four known compounds $2,3,4$ and 5 were identified as 5,7,3',4'-tetrahydroxy-6- C- $\beta$-L-arabinosyl flavonoside(2), 5,7,4'-trihydroxy6-C- $\beta$-D-glucopyranosyl flavonoside(3), 5,7,3', 4'- tetrahydroxy-6-C$\beta$-D-glucopyranosyl flavonoside(4) and $5,7,3^{\prime}, 4^{\prime}$ '-tetrahydroxy- 8 -C- $\beta$ D-glucopyranosyl flavonoside(5), respectively, by comparison of their spectroscopic data with the literature ${ }^{13,14}$.

\section{ACKNOWLEDGEMENTS}

This work is supported by the National Science and Technology Infrastructure Program (No.2006BAD19B08); National Natural Science Foundation of China (30871961) and Basic Science Research Fund Program of the International Centre for Bamboo and Rattan (ICBR) (8618-8).

\section{REFERENCES}

1. M.G. Hertog, D. Kromhout, C. Arayanis, H. Blackburn, R. Buzina, F. Fidanza, S. Giampaoli, A. Jansen, A. Menotti, and S. Nedeljkovic, Arch. Intern.Med. 155, 381, (1995).

2. L. Yochum, L.H. Kushi, K. Meyer, and A.R. Folsom, Am. J. Epidemiol. 149, 943, (1999).

3. P. Knekt, R. Jarvinen, A. Reunanen, and J. Maatela, Br. Med. J. 312, 478, (1996).

4. L.L. Marchand, S.P. Murphy, J.H. Hankin, L,R. Wilkens, and L.N. colonel, J.Natl, cancer Inst.92, 154, (2000).

5. H. Madari, D. Panda, L. Wilson, Cancer Res. 63, 6, 1214-1220, (2003).

6. Y. Zhang. B.L. Bao. B.Y. Lu. Y.P. Ren. X.W. Tie. J. Chromatogr. A. 1065, 177, (2005).

7. J. Burns. P.T. Gardner. J.O. Neil. S. Crawford. J. Agric.Food Chem. 48, $220,(2000)$

8. T. Kaneko. N. Baba, Biosci. Biotechnol Biochem. 63, 323, (1999).

9. X.M. Hu. Zhonghuabencao Pharmacopoeia, Vol 23 , (People's Medical Publishing House, Beijing, 1999), (Chinese) P.323-324.

10. J. Sun, Y.D. Yue, F. Tang, X.F. Guo. J.Asian Nat.Prod.Res. 12, 248. (2010).

11. N. Wang. J.H. Wang. X.Li. Ling. J.Asian Nat.Prod.Res. 8, 753, (2006),

12. Z.G. Zheng. R.H. Liu. L.Y. Kong. Chin. Chem. Lett. 17, 919, (2006).

13. H. Geiger, Mabry T. J. Tetrahedron. 34, 1387. (1978)

14. P. C. Zhang. The Chemistry of Flavonoid (Chemical Industry Press, Beijing, 2009), (Chinese) P.85-93. 